\title{
Asymptotic Behavior of Solutions for Semilinear Volterra Diffusion Equations with Spatial Inhomogeneity and Advection
}

\author{
Yusuke YOSHIDA and Yoshio YAMADA \\ Waseda University \\ (Communicated by T. Iguchi)
}

\begin{abstract}
This paper is concerned with semilinear Volterra diffusion equations with spatial inhomogeneity and advection. We intend to study the effects of interaction among diffusion, advection and Volterra integral under spatially inhomogeneous environments. Since the existence and uniqueness result of global-in-time solutions can be proved in the standard manner, our main interest is to study their asymptotic behavior as $t \rightarrow \infty$. For this purpose, we study the related stationary problem by the monotone method and establish some sufficient conditions on the existence of a unique positive solution. Its global attractivity is also studied with use of a suitable Lyapunov functional.
\end{abstract}

\section{Introduction}

In the present paper we consider a spatially inhomogeneous logistic equation with diffusion, advection and continuous delay represented in the form of a Volterra integral. Let $\Omega$ be a bounded domain in $\mathbf{R}^{N}$ with smooth boundary $\partial \Omega$. Let $u=u(x, t)$ for $x \in \Omega$ and $t \geq 0$ satisfy

$$
\begin{cases}u_{t}=\operatorname{div}\{d(x) \nabla u-u \Gamma(x)\}+u\{a(x)-b(x) u-c(x) k * u(t)\} \\ B u=0 & \text { in } \Omega \times(0, \infty), \\ u(\cdot, 0)=u_{0} & \text { on } \partial \Omega \times(0, \infty),\end{cases}
$$

where $a, b$ and $c$ are functions of class $L^{\infty}(\Omega)$ with $b \geq 0$ and $c \geq 0$ in $\Omega$. A diffusion coefficient $d$ is a positive function of class $C^{1+\alpha}(\bar{\Omega})$ with $\alpha \in(0,1)$ and $\Gamma$ is a function of the form

$$
\Gamma(x)=d(x) \nabla \gamma(x)
$$

Received March 18, 2015; revised August 31, 2015

2010 Mathematics Subject Classification: 35B40 (Primary), 35J61, 35K57, 35R09, 92D25 (Secondary)

Key words and phrases: Spatial inhomogeneity, logistic equation, Volterra diffusion equation, advection, global attractivity, Lyapunov functional 
with $\gamma \in C^{2+\alpha}(\bar{\Omega})$. Moreover, $k * u$ is defined by

$$
k * u(t)=\int_{0}^{t} k(t-s) u(s) d s,
$$

where $k$ is a nonnegative function of class $C^{1}(0, \infty) \cap L^{1}(0, \infty)$ satisfying

$$
\int_{0}^{\infty} k(t) d t=1 .
$$

Boundary operator $B$ represents the following boundary condition

$$
B u=u \quad \text { or } \quad B u=\partial u / \partial n+\{\beta(x)-(\partial \gamma / \partial n)\} u,
$$

where $\partial / \partial n$ denotes the outward normal derivative to $\partial \Omega$ and $\beta$ is a nonnegative function of class $C^{1+\alpha}(\partial \Omega)$.

Our problem (1.1) appears in population biology and $u$ denotes the population density of a biological species. When $\Gamma$ is given by (1.2), flux $d u \nabla \gamma$ describes the movement of each individual species toward more favorable habitat where $\gamma$ is large. Throughout this paper, we always assume that

(A.1) $u_{0}$ is a nonnegative (not identically zero) function of class $L^{\infty}(\Omega)$,

(A.2) $\inf _{x \in \Omega}\{b(x)+c(x)\}>0$.

If $c \equiv 0$ and $\gamma$ is a constant function, then (1.1) is an initial boundary value problem for a spatially inhomogeneous logistic diffusion equation and the dynamics of solutions of (1.1) is well known (see the papers and the monograph of Cantrell-Cosner $[8,9,10]$ ). Furthermore, for the case $\gamma=\varepsilon a$ with $\varepsilon>0$ and $a \in C^{2+\alpha}(\bar{\Omega})$, see, e.g., [3, 5, 10, 11, 15, 16]. However, it is more realistic to take account of effects of time delay in the study of population biology. The term $k * u$ is introduced to describe effects from the past to the present. The following two functions $k$ are typical delay kernels in mathematical biology:

$$
\begin{gathered}
k(t)=(1 / T) e^{-t / T}, \\
k(t)=\left(t / T^{2}\right) e^{-t / T} .
\end{gathered}
$$

Here (K.1) and (K.2) are called a weak delay kernel and a strong delay kernel, respectively. For instance, they appear in the bacteria model (for details, see Iida [14]).

Our purpose of the present paper is to study

(a) Existence and uniqueness of global solutions of (1.1),

(b) Asymptotic behavior of solutions as $t \rightarrow \infty$,

(c) Existence, uniqueness and stability of positive stationary solutions.

When $a, b, c, d$ and $\gamma$ are constants, (a)-(c) have been studied by many authors and lots of results have been obtained (see [14, 19, 23, 26, 27] with homogeneous Neumann boundary condition and [24, 29] with homogeneous Dirichlet boundary condition). In addition, some systems of Volterra diffusion equations have been also studied in [1, 28]. However, it seems 
that there are few works for Volterra diffusion equations under inhomogeneous environment. So we intend to study Volterra diffusion equations with spatial inhomogeneity.

Before studying (1.1), we will use a change of variables $v=e^{-\gamma(x)} u$, which allows to rewrite (1.1) with (1.2) as follows:

$$
\begin{cases}v_{t}=\operatorname{div}\{d(x) \nabla v\}+d(x) \nabla \gamma \cdot \nabla v+v\left\{a(x)-b(x) e^{\gamma(x)} v\right. & \left.-c(x) e^{\gamma(x)} k * v(t)\right\} \\ B^{\prime} v=0 & \text { in } \Omega \times(0, \infty), \\ v(\cdot, 0)=v_{0} & \text { on } \partial \Omega \times(0, \infty),\end{cases}
$$

where $v_{0}=e^{-\gamma(x)} u_{0}$ and

$$
B^{\prime} v=v \quad \text { or } \quad B^{\prime} v=\partial v / \partial n+\beta(x) v .
$$

Hereafter we will study (P) instead of (1.1). A stationary problem associated with (P) is given by

$$
\begin{cases}\operatorname{div}\{d(x) \nabla \varphi\}+d(x) \nabla \gamma \cdot \nabla \varphi+\varphi\left[a(x)-\{b(x)+c(x)\} e^{\gamma(x)} \varphi\right]=0 & \text { in } \Omega, \\ B^{\prime} \varphi=0 & \text { on } \partial \Omega,\end{cases}
$$

(note (1.3)).

Our first task is to show the existence and uniqueness of a global solution of $(\mathrm{P})$. We will discuss it in the framework of $L^{p}(\Omega)$ theory. The next step is to study the asymptotic behavior of the solution as $t \rightarrow \infty$. The asymptotic behavior is very closely related with the stationary problem (SP). Since (SP) is a semilinear elliptic problem with logistic term, the standard monotone method enables us to show the existence and uniqueness result of positive solution $\varphi$. Here it should be noted that $a$ is not necessarily a positive function. So we will give sufficient conditions on $a$ and $d$ which assure the existence of a unique positive solution $\varphi$.

Once the existence of a positive solution of (SP) is established, the subsequent problem is to study its stability. If there is no delay term, then the analytic procedure to discuss the stability of $\varphi$ is well established. However, in case of the presence of the delay term, one cannot apply such a method because the comparison principle does not hold for (P). So we will introduce a suitable Lyapunov functional to study the global stability of $\varphi$ (see (4.5)). Then we will show some sufficient conditions for the global attractivity of $\varphi$ with use of the Laplace transform of $k$.

The plan of this paper is as follows. In Section 2 we will give the existence and uniqueness of global solutions of $(\mathrm{P})$. In Section 3 we will show the existence and uniqueness of a positive solution of (SP) and some sufficient conditions for the existence. Our main results are concerned with asymptotic behavior of solutions for $(\mathrm{P})$ as $t \rightarrow \infty$ and they are contained in Sections 4 and 5. In these sections we will show some sufficient conditions for the global attractivity of the positive stationary solution by separating arguments into two cases: $\inf _{x \in \Omega} b(x)>0$ and $\inf _{x \in \Omega} b(x)=0$. 
Notation. For $p \in[1, \infty), L^{p}(\Omega)$ denotes the usual Banach space with norm $\|\cdot\|_{p, \Omega}$ and $L^{\infty}(\Omega)$ denotes the space of essentially bounded and measurable functions with norm $\|\cdot\|_{\infty, \Omega}$. If there is no confusion, we will omit the subscript $\Omega$. For each $p \in[1, \infty)$ and integer $k \in[1, \infty), W^{k, p}(\Omega)$ denotes the usual Sobolev space of measurable functions $u: \Omega \rightarrow \mathbf{R}$ such that $u$ and its distributional derivatives up to order $k$ belong to $L^{p}(\Omega)$. Its norm is defined by

$$
\|u\|_{k, p, \Omega}=\left(\sum_{|\alpha| \leq k}\left\|D^{\alpha} u\right\|_{p}^{p}\right)^{1 / p},
$$

where $\alpha$ denotes a multi-index for derivatives. If there is no confusion, then we will also omit the subscript $\Omega$. We sometimes write $H^{k}(\Omega)$ instead of $W^{k, 2}(\Omega)$. Moreover, $W_{0}^{k, p}(\Omega)$ denotes the closure of $C_{0}^{\infty}(\Omega)$ with respect to $W^{k, p}(\Omega)$-norm. In the same way as $H^{k}(\Omega)$ we sometimes write $H_{0}^{k}(\Omega)$ instead of $W_{0}^{k, 2}(\Omega)$.

\section{Existence and uniqueness of global solutions}

In this section we will study (P) in the framework of $L^{p}(\Omega)$-space with $p>1$. Define a closed linear operator $A$ with dense domain $D(A)$ by

$$
A v=-\operatorname{div}\{d(x) \nabla v\}-d(x) \nabla \gamma \cdot \nabla v
$$

and

$$
D(A)= \begin{cases}W_{0}^{1, p}(\Omega) \cap W^{2, p}(\Omega) & \text { if } B^{\prime} v=v, \\ \left\{v \in W^{2, p}(\Omega) \mid B^{\prime} v=0 \text { on } \partial \Omega\right\} & \text { if } B^{\prime} v=\partial v / \partial n+\beta(x) v .\end{cases}
$$

For each $\mu \in[0,1]$, we introduce the fractional power space $D\left(A^{\mu}\right)$ equipped with the graph norm of $A^{\mu}$ in the standard manner. If $p>\max \{1, N / 2\}$, then it is known that

$$
D\left(A^{\mu}\right) \subset C^{v}(\bar{\Omega}) \quad \text { with } v \in[0,2 \mu-(N / p)),
$$

(see [12] or [17]). It is also well known that $-A$ generates an analytic semigroup $\left\{e^{-t A}\right\}_{t \geq 0}$ in $L^{p}(\Omega)$.

We will write $(\mathrm{P})$ as

$$
\left\{\begin{array}{l}
v_{t}+A v=F(x, v, k * v(t)) \\
v(0)=v_{0}
\end{array}\right.
$$

where

$$
F(x, v, w)=v\left\{a(x)-b(x) e^{\gamma(x)} v-c(x) e^{\gamma(x)} w\right\} .
$$

Let $v$ be a local solution of (P) in $\Omega \times\left[0, T_{0}\right]$ with some $T_{0}>0$. In order to extend such a local solution of $(\mathrm{P})$, we are led to consider the existence of solutions of the following initial 
value problem:

$$
\left\{\begin{array}{l}
w_{t}+A w=w\left\{\tilde{a}(x, t)-b(x) e^{\gamma(x)} w-c(x) e^{\gamma(x)} k * w(t)\right\}, \\
w(0)=v\left(T_{0}\right)
\end{array}\right.
$$

with

$$
\tilde{a}(x, t)=a(x)-c(x) e^{\gamma(x)} \int_{0}^{T_{0}} k\left(t+T_{0}-s\right) v(s) d s .
$$

Hence it will be better to study the local existence problem in the following more general form in place of (2.2):

$$
\left\{\begin{array}{l}
v_{t}+A v=F^{*}(x, t, v, k * v(t)) \\
v(0)=v_{0}
\end{array}\right.
$$

where

$$
F^{*}(x, t, v, w)=v\left\{\tilde{a}(x, t)-b(x) e^{\gamma(x)} v-c(x) e^{\gamma(x)} w\right\} .
$$

We can prove the following local existence result:

Proposition 2.1. Let $p>\max \{1, N / 2\}$ and assume $\tilde{a} \in C^{\vartheta}\left([0, T] ; L^{\infty}(\Omega)\right)$ with $\vartheta \in(0,1)$ and $v_{0} \in L^{p}(\Omega)$. Then there exists a positive number $T_{0} \leq T$ such that (2.3) has a unique solution $v$ in the class

$$
v \in C\left(\left[0, T_{0}\right] ; L^{p}(\Omega)\right) \cap C^{1}\left(\left(0, T_{0}\right] ; L^{p}(\Omega)\right) \cap C\left(\left(0, T_{0}\right] ; D(A)\right) .
$$

PROOF. By variation of constant formula, (2.3) can be rewritten in the form of an integral equation

$$
v(t)=e^{-t A} v_{0}+\int_{0}^{t} e^{-(t-s) A} F^{*}(s, v(s), k * v(s)) d s .
$$

Applying Banach's fixed point theorem to (2.4), we can prove the local existence of a solution of (2.3). For details, see for instance [13].

We can also establish the global existence theorem.

THEOREM 2.1. Let $p>\max \{1, N / 2\}$. Then $(\mathrm{P})$ has a unique solution $v$ in the class

$$
v \in C\left([0, \infty) ; L^{p}(\Omega)\right) \cap C^{1}\left((0, \infty) ; L^{p}(\Omega)\right) \cap C((0, \infty) ; D(A)) ;
$$

which satisfies

$$
v>0 \quad \text { in } \Omega \times(0, \infty) \text { and } \partial v / \partial n<0 \quad \text { on } \partial \Omega \times(0, \infty)
$$

if $B^{\prime} v=v$, and

$$
v>0 \text { in } \bar{\Omega} \times(0, \infty)
$$


if $B^{\prime} v=\partial v / \partial n+\beta(x) v$. Moreover, if $\inf _{x \in \Omega} b(x)>0$, then

$$
v \leq m \text { in } \Omega \times(0, \infty),
$$

where

$$
m=\max \left\{\left\|v_{0}\right\|_{\infty}, \sup _{x \in \Omega} \frac{a(x)}{b(x) e^{\gamma(x)}}\right\} .
$$

PROOF. Since $v_{0} \geq 0$, it is easy to show by the maximum principle ([18]) that $v(\cdot, t) \geq$ 0 . By virtue of the nonnegativity of $k, v$ satisfies

$$
v_{t} \leq \operatorname{div}\{d(x) \nabla v\}+d(x) \nabla \gamma \cdot \nabla v+v\left\{a(x)-b(x) e^{\gamma(x)} v\right\} .
$$

If $\inf _{x \in \Omega} b(x)>0$, then the comparison theorem (see, e.g., [25]) implies $v \leq m$. Finally, the strong maximum principle [18] shows (2.5) and (2.6). Hence in view of Proposition 2.1, we can obtain the existence and uniqueness of a global solution of $(\mathrm{P})$.

\section{Positive stationary solutions}

In this section we will look for positive solutions of (SP). Consider the following eigenvalue problem:

$$
\begin{cases}-\operatorname{div}\{d(x) \nabla \psi\}-d(x) \nabla \gamma \cdot \nabla \psi-a(x) \psi=\lambda \psi & \text { in } \Omega, \\ B^{\prime} \psi=0 & \text { on } \partial \Omega .\end{cases}
$$

Note that the first equation of (EP) can be rewritten by multiplying $e^{\gamma(x)}$ to both sides as

$$
-\operatorname{div}\left\{d(x) e^{\gamma(x)} \nabla \psi\right\}-a(x) e^{\gamma(x)} \psi=\lambda e^{\gamma(x)} \psi .
$$

Let $\lambda_{1} \equiv \lambda_{1}(a, d, \gamma)$ denote the principal eigenvalue of (EP). Then it is given by the following variational characterization:

$$
\lambda_{1}=\inf _{\substack{\psi \in H^{1}(\Omega) \\ \psi \neq 0}} \frac{\int_{\Omega} d(x) e^{\gamma(x)}|\nabla \psi|^{2} d x+\int_{\partial \Omega} d(x) \beta(x) e^{\gamma(x)} \psi^{2} d \sigma-\int_{\Omega} a(x) e^{\gamma(x)} \psi^{2} d x}{\int_{\Omega} e^{\gamma(x)} \psi^{2} d x}
$$

if $B^{\prime} \psi=\partial \psi / \partial n+\beta(x) \psi$, while

$$
\lambda_{1}=\inf _{\substack{\psi \in H_{0}^{1}(\Omega) \\ \psi \neq 0}} \frac{\int_{\Omega} d(x) e^{\gamma(x)}|\nabla \psi|^{2} d x-\int_{\Omega} a(x) e^{\gamma(x)} \psi^{2} d x}{\int_{\Omega} e^{\gamma(x)} \psi^{2} d x}
$$

if $B^{\prime} \psi=\psi$. Then we are ready to give the existence, nonexistence and uniqueness results of a positive solution of (SP). 
THEOREM 3.1. Problem (SP) has a positive solution $\varphi$ if and only if $\lambda_{1}<0$, where $\lambda_{1}$ is the principal eigenvalue of (EP). Moreover, when $\varphi$ exists, it is uniquely determined and it satisfies

$$
0<\varphi \leq M \quad \text { in } \Omega
$$

and

$$
\begin{cases}\partial \varphi / \partial n<0 & \text { on } \partial \Omega \text { if } B^{\prime} \varphi=\varphi \\ 0<\varphi \leq M & \text { on } \partial \Omega \text { if } B^{\prime} \varphi=\partial \varphi / \partial n+\beta(x) \varphi\end{cases}
$$

where

$$
M=\sup _{x \in \Omega} \frac{a(x)}{\{b(x)+c(x)\} e^{\gamma(x)}} .
$$

REMARK 3.1. Since $\lambda_{1}<0$ requires $\sup _{x \in \Omega} a(x)>0, M$ is a positive number.

PROOF. Recall (3.1). Then the first equation of (SP) can be rewritten as

$$
\operatorname{div}\left\{d(x) e^{\gamma(x)} \nabla \varphi\right\}+e^{\gamma(x)} \varphi\left[a(x)-\{b(x)+c(x)\} e^{\gamma(x)} \varphi\right]=0 .
$$

Take $\bar{\varphi}=\bar{M}$ with positive number $\bar{M} \geq M$ and $\underline{\varphi}=\varepsilon \psi_{1}$ with small positive number $\varepsilon$, where $\psi_{1}$ is an eigenfunction corresponding to $\lambda_{1}$ such that $\sup _{x \in \Omega} \psi_{1}(x)=1$. It is easy to prove that $\bar{\varphi}$ and $\underline{\varphi}$ become a pair of upper and lower solutions to (3.4). Therefore, the monotone method (see Amann [2] or Sattinger [22]) assures the existence of a positive solution $\varphi$ of (SP) satisfying $\underline{\varphi} \leq \varphi \leq \bar{\varphi}$. For the uniqueness of $\varphi$, see for instance the paper of Berestycki [4]. Finally, note that (3.3) comes from the strong maximum principle [18].

We can show the following result on the asymptotic behavior of solutions for $(\mathrm{P})$ in case $\lambda_{1} \geq 0$, where (SP) has no positive solution:

THEOREM 3.2. Assume

$$
\inf _{x \in \Omega} b(x)>0 \quad \text { and } \quad \lambda_{1} \geq 0 \quad \text { or } \quad \inf _{x \in \Omega} b(x)=0 \quad \text { and } \quad \lambda_{1}>0 .
$$

Then every solution $v$ of $(\mathrm{P})$ satisfies

$$
\lim _{t \rightarrow \infty} v(t)=0 \quad \text { uniformly in } \bar{\Omega} .
$$

PROOF. Since $c$ and $k$ are nonnegative, the positivity of $v$ implies

$$
v_{t} \leq \operatorname{div}\{d(x) \nabla v\}+d(x) \nabla \gamma \cdot \nabla v+v\left\{a(x)-b(x) e^{\gamma(x)} v\right\} .
$$


Consider the following problem:

$$
\begin{cases}w_{t}=\operatorname{div}\{d(x) \nabla w\}+d(x) \nabla \gamma \cdot \nabla w+w\left\{a(x)-b(x) e^{\gamma(x)} w\right\} & \text { in } \Omega \times(0, \infty), \\ B^{\prime} w=0 & \text { on } \partial \Omega \times(0, \infty), \\ w(\cdot, 0)=\left\|v_{0}\right\|_{\infty} & \text { in } \Omega .\end{cases}
$$

Owing to (3.5), the theory of dynamical systems enables us to yield

$$
\lim _{t \rightarrow \infty} w(t)=0 \quad \text { uniformly in } \bar{\Omega} .
$$

Since $v \leq w$ by the comparison theorem, the conclusion easily follows.

By virtue of Theorem 3.1 we will search some sufficient conditions on $a, d$ for $\lambda_{1}<0$, which assures the existence of a positive stationary solution of (SP). Set

$$
\Omega^{+}=\{x \in \Omega \mid a(x)>0\},
$$

and assume $\Omega^{+} \neq \emptyset$ in this section. Consider the following eigenvalue problem:

$$
\begin{cases}-\operatorname{div}\{d(x) \nabla \rho\}-d(x) \nabla \gamma \cdot \nabla \rho=\mu a(x) \rho & \text { in } \Omega, \\ B^{\prime} \rho=0 & \text { on } \partial \Omega .\end{cases}
$$

Similarly as in (EP), the first equation of (3.7) can be rewritten as

$$
-\operatorname{div}\left\{d(x) e^{\gamma(x)} \nabla \rho\right\}=\mu a(x) e^{\gamma(x)} \rho .
$$

Let $\mu_{1}^{+} \equiv \mu_{1}^{+}(a, d, \gamma)$ denote the positive principal eigenvalue of (3.7). It is given by the following variational characterization (see the monograph of Cantrell-Cosner [10, Theorem 2.4]):

$$
\frac{1}{\mu_{1}^{+}}=\sup _{\substack{\rho \in H^{1}(\Omega) \\ \rho \neq 0}} \frac{\int_{\Omega} a(x) e^{\gamma(x)} \rho^{2} d x}{\int_{\Omega} d(x) e^{\gamma(x)}|\nabla \rho|^{2} d x+\int_{\partial \Omega} d(x) \beta(x) e^{\gamma(x)} \rho^{2} d \sigma}
$$

if $B^{\prime} \psi=\partial \psi / \partial n+\beta(x) \psi$, while

$$
\frac{1}{\mu_{1}^{+}}=\sup _{\substack{\rho \in H_{0}^{1}(\Omega) \\ \rho \neq 0}} \frac{\int_{\Omega} a(x) e^{\gamma(x)} \rho^{2} d x}{\int_{\Omega} d(x) e^{\gamma(x)}|\nabla \rho|^{2} d x}
$$

if $B^{\prime} \psi=\psi$. In (3.8) and (3.9), $\int_{\Omega} a(x) e^{\gamma(x)} \rho^{2} d x>0$ for suitable $\rho \in H^{1}(\Omega)$ because $\Omega^{+}$ is non-empty. So $\mu_{1}^{+}>0$ can be defined in case $B^{\prime} \psi=\psi$ or $B^{\prime} \psi=\partial \psi / \partial n+\beta \psi$ with $\beta \geq 0(\beta \not \equiv 0)$. In case $\beta \equiv 0$ the denominator in the right-hand side of (3.8) vanishes if 
$\rho$ is a constant function. Therefore, in case $B^{\prime} \psi=\partial \psi / \partial n$, the existence of positive $\mu_{1}^{+}$is a delicate problem. Actually, we can show that $\mu_{1}^{+}$exists if and only if

$$
\int_{\Omega} a(x) e^{\gamma(x)} d x<0
$$

(see Proposition A in Appendix). The relation between $\lambda_{1}$ and $\mu_{1}^{+}$is given by the following proposition (see [3, Proposition 2.2] or [10, Theorem 2.6]):

Proposition 3.1. Let $\lambda_{1}$ be the principal eigenvalue of (EP) and let $\mu_{1}^{+}$be the positive principal eigenvalue of (3.7).

(i) Let $B^{\prime} \psi=\psi$ or $B^{\prime} \psi=\partial \psi / \partial n+\beta(x) \psi$ with $\beta \not \equiv 0$. Then $\lambda_{1}$ is negative if and only if $\mu_{1}^{+}<1$.

(ii) Let $B^{\prime} \psi=\partial \psi / \partial n$. When (3.10) holds, $\lambda_{1}$ is negative if and only if $\mu_{1}^{+}<1$; while $\lambda_{1}$ is always negative when

$$
\int_{\Omega} a(x) e^{\gamma(x)} d x>0
$$

Making use of Proposition 3.1 we will prove the following result:

Proposition 3.2. Define $\Omega^{+}$by (3.6). Let $B^{\prime} \psi=\psi$ or $B^{\prime} \psi=\partial \psi / \partial n$ with (3.10) or $B^{\prime} \psi=\partial \psi / \partial n+\beta(x) \psi$ with $\beta \not \equiv 0$. Then there exists a positive constant $d^{*}$ such that $\lambda_{1}<0$ for any $d$ satisfying $\|d\|_{\infty, \Omega^{+}}<d^{*}$.

PROOF. We will only discuss the case $B^{\prime} \psi=\psi$. The other case can be handled similarly. Take any connected set $\Omega^{*} \subset \Omega^{+}$. Take any function $\rho \in H_{0}^{1}\left(\Omega^{*}\right)$ and let $\tilde{\rho}: \Omega^{*} \rightarrow \mathbf{R}$ be the natural extension of $\rho$. It follows from (3.9) that

$$
\begin{aligned}
\frac{1}{\mu_{1}^{+}} \geq \sup _{\substack{\rho \in H_{0}^{1}\left(\Omega^{*}\right) \\
\rho \neq 0}} \frac{\int_{\Omega} a(x) e^{\gamma(x)} \tilde{\rho}^{2} d x}{\int_{\Omega} d(x) e^{\gamma(x)}|\nabla \tilde{\rho}|^{2} d x} \\
\geq\|d\|_{\infty, \Omega^{*}}^{-1} \sup _{\substack{\rho \in H_{0}^{1}\left(\Omega^{*}\right) \\
\rho \neq 0}} \frac{\int_{\Omega^{*}} a(x) e^{\gamma(x)} \rho^{2} d x}{\int_{\Omega^{*}} e^{\gamma(x)}|\nabla \rho|^{2} d x} .
\end{aligned}
$$

Define $d^{*}$ by

$$
d^{*}=\sup _{\substack{\rho \in H_{0}^{1}\left(\Omega^{*}\right) \\ \rho \neq 0}} \frac{\int_{\Omega^{*}} a(x) e^{\gamma(x)} \rho^{2} d x}{\int_{\Omega^{*}} e^{\gamma(x)}|\nabla \rho|^{2} d x} .
$$


Then $\mu_{1}^{+}<1$ for any $d$ satisfying $\|d\|_{\infty, \Omega^{*}}<d^{*}$. Therefore, Proposition 3.1 yields the conclusion.

Propositions 3.1 and 3.2 imply that a positive stationary solution exists if a diffusion coefficient is sufficiently small in a favorable habitat $\Omega^{+}$or (3.11) is achieved with homogeneous Neumann boundary condition. In ecology, this fact asserts that there is a chance for a species to survive if the species stays in a favorable habitat $\Omega^{+}$.

REMARK 3.2. Assume $a \in C^{2+\alpha}(\bar{\Omega})$ and $\gamma=C a$. Then we can easily obtain (3.11) if $C$ is sufficiently large. This implies that a sufficiently strong advection toward more favorable habitat gives a chance for the species to survive. For details, see Belgacem-Cosner [3, Theorem 2.4].

\section{Global attractivity of $\varphi$ in case $\inf _{x \in \Omega} b(x)>0$}

In this section we consider the case $\inf _{x \in \Omega} b(x)>0$ and $\lambda_{1}<0$, which assures that there exists a unique positive solution $\varphi$ of (SP). Denote by $\hat{k}$ the Laplace transform of $k$ :

$$
\hat{k}(\lambda)=\int_{0}^{\infty} e^{-\lambda t} k(t) d t .
$$

Then we can prove the global attractivity of $\varphi$ in the following manner:

THEOREM 4.1. Assume $\inf _{x \in \Omega} b(x)>0, \lambda_{1}<0$ and $t k \in L^{1}(0, \infty)$. Furthermore, assume that there exists a positive constant $k_{0}$ such that

$$
b(x)+\operatorname{Re} \hat{k}(i \eta) c(x) \geq k_{0} \quad \text { for } x \in \Omega \text { and } \eta \in \mathbf{R} .
$$

Then every solution $v$ of $(\mathrm{P})$ satisfies

$$
\lim _{t \rightarrow \infty} v(t)=\varphi \quad \text { uniformly in } \bar{\Omega} .
$$

REMARK 4.1. Both kernels (K.1) and (K.2) satisfy $t k \in L^{1}(0, \infty)$. Moreover, for (K.1),

$$
\inf _{\eta \in \mathbf{R}} \operatorname{Re} \hat{k}(i \eta)=\inf _{\eta \in \mathbf{R}} \operatorname{Re}\left(\frac{1}{1+i \eta T}\right)=0,
$$

and for (K.2),

$$
\inf _{\eta \in \mathbf{R}} \operatorname{Re} \hat{k}(i \eta)=\inf _{\eta \in \mathbf{R}} \operatorname{Re}\left(\frac{1}{1+i \eta T}\right)^{2}=-\frac{1}{8} .
$$

From (4.3) and (4.4), $\varphi$ is always globally attractive for (K.1), while it is globally attractive for (K.2) if

$$
\inf _{x \in \Omega}\left\{b(x)-\frac{c(x)}{8}\right\}>0 .
$$


When we consider (P) with spatially homogeneous coefficients, constant function $\gamma$ and homogeneous Neumann boundary condition, it follows from the result of Yamada [27] that, if $k$ is given by (K.2), then $\varphi$ loses its stability and periodic solutions bifurcate from $\varphi$ under a suitable situation.

To prove Theorem 4.1, we will follow the arguments used in [29]. Let $\varphi$ be a positive solution of (SP). We introduce the following nonnegative functional:

$$
\begin{aligned}
E(v) & =\int_{\Omega} e^{\gamma(x)} \varphi^{2}(x) g(v(x) / \varphi(x)) d x \\
& =\int_{\Omega} e^{\gamma(x)} \varphi(x)\left\{v(x)-\varphi(x)-\varphi(x) \log \frac{v(x)}{\varphi(x)}\right\} d x,
\end{aligned}
$$

where

$$
g(v)=v-1-\log v .
$$

When $\gamma$ is a constant, this functional has been used in [1] and [29].

REMARK 4.2. It should be noted that (4.5) is well defined on $\partial \Omega$ under homogeneous Dirichlet boundary condition. Indeed, we see from (2.5) and (3.3)

$$
\lim _{x \rightarrow x_{0}} \frac{v(x, t)}{\varphi(x)}=\frac{\partial v\left(x_{0}, t\right)}{\partial n}\left\{\frac{\partial \varphi\left(x_{0}\right)}{\partial n}\right\}^{-1}>0 \text { for }\left(x_{0}, t\right) \in \partial \Omega \times(0, \infty) .
$$

Hence it is possible to define $v / \varphi$ on $\partial \Omega$ in this sense.

We will prepare the following key lemma:

Lemma 4.1 (cf. [29, Lemma 3.1]). Define E(v) by (4.5). Then any solution $v$ of $(\mathrm{P})$ satisfies

$$
\begin{aligned}
\frac{d}{d t} E(v(t))= & -\int_{\Omega} d(x) e^{\gamma(x)} \varphi^{2}\left|\nabla\left\{\log \frac{v(t)}{\varphi}\right\}\right|^{2} d x \\
& -\int_{\Omega} b(x) e^{2 \gamma(x)} \varphi\{v(t)-\varphi\}^{2} d x \\
& -\int_{\Omega} c(x) e^{2 \gamma(x)} \varphi\{v(t)-\varphi\} k *(v-\varphi)(t) d x \\
& +\int_{t}^{\infty} k(s) d s \int_{\Omega} c(x) e^{2 \gamma(x)} \varphi^{2}\{v(t)-\varphi\} d x
\end{aligned}
$$

PROOF. We will only prove the case $B^{\prime} v=v$ because the proof of the other case is 
essentially the same. Differentiation of (4.5) with respect to $t$ yields

$$
\begin{aligned}
\frac{d}{d t} E(v(t))= & \int_{\Omega} e^{\gamma(x)}\left\{1-\frac{\varphi}{v(t)}\right\} v_{t}(t) \varphi d x \\
= & \int_{\Omega} e^{\gamma(x)} \varphi\left\{1-\frac{\varphi}{v(t)}\right\}[\operatorname{div}\{d(x) \nabla v(t)\}+d(x) \nabla \gamma \cdot \nabla v] d x \\
& +\int_{\Omega} e^{\gamma(x)} \varphi\{v(t)-\varphi\}\left\{a(x)-b(x) e^{\gamma(x)} v(t)-c(x) e^{\gamma(x)} k * v(t)\right\} d x
\end{aligned}
$$

Similarly to (3.1), note

$$
e^{\gamma(x)}[\operatorname{div}\{d(x) \nabla v(t)\}+d(x) \nabla \gamma \cdot \nabla v]=\operatorname{div}\left\{d(x) e^{\gamma(x)} \nabla v(t)\right\} .
$$

In view of (1.3),

$$
\begin{aligned}
& a(x)-b(x) e^{\gamma(x)} v-c(x) e^{\gamma(x)} k * v(t) \\
= & a(x)-\{b(x)+c(x)\} e^{\gamma(x)} \varphi-b(x) e^{\gamma(x)}(v-\varphi)-c(x) e^{\gamma(x)} k *(v-\varphi)(t) \\
& +c(x) e^{\gamma(x)} \varphi \int_{t}^{\infty} k(s) d s .
\end{aligned}
$$

Since $\varphi$ is a solution of (SP), it follows that

$$
\begin{aligned}
\frac{d}{d t} E(v(t))= & \int_{\Omega} \varphi\left\{1-\frac{\varphi}{v(t)}\right\} \operatorname{div}\left\{d(x) e^{\gamma(x)} \nabla v(t)\right\} d x \\
& -\int_{\Omega}\{v(t)-\varphi\} \operatorname{div}\left\{d(x) e^{\gamma(x)} \nabla \varphi\right\} d x-\int_{\Omega} b(x) e^{2 \gamma(x)} \varphi\{v(t)-\varphi\}^{2} d x \\
& -\int_{\Omega} c(x) e^{2 \gamma(x)} \varphi\{v(t)-\varphi\} k *(v-\varphi)(t) d x \\
& +\int_{t}^{\infty} k(s) d s \int_{\Omega} c(x) e^{2 \gamma(x)} \varphi^{2}\{v(t)-\varphi\} d x .
\end{aligned}
$$

Moreover, the integration by parts yields

$$
\begin{aligned}
\int_{\Omega} \varphi & \left\{1-\frac{\varphi}{v(t)}\right\} \operatorname{div}\left\{d(x) e^{\gamma(x)} \nabla v(t)\right\} d x-\int_{\Omega}\{v(t)-\varphi\} \operatorname{div}\left\{d(x) e^{\gamma(x)} \nabla \varphi\right\} d x \\
= & -\int_{\Omega} d(x) e^{\gamma(x)}\left\{|\nabla \varphi|^{2}-\frac{2 \varphi}{v(t)} \nabla \varphi \cdot \nabla v(t)-\frac{\varphi^{2}}{v^{2}(t)}|\nabla v(t)|^{2}\right\} d x \\
= & -\int_{\Omega} d(x) e^{\gamma(x)} \varphi^{2}\left|\nabla\left\{\log \frac{v(t)}{\varphi}\right\}\right|^{2} d x .
\end{aligned}
$$

Therefore, (4.7) follows.

We will also prepare some regularity results. 
LEMMA 4.2. Let $v$ be a bounded solution of $(\mathrm{P})$ and let $\delta$ be any positive number. Then there exist positive constants $K_{1}, K_{2}$ and $K_{3}$ such that for $p>1$ and $\mu \in[0,1)$,

$$
\sup _{t \geq \delta}\left\|A^{\mu} v(t)\right\|_{p} \leq K_{1}
$$

and for any $h>0$,

$$
\sup _{t \geq \delta}\left\|A^{\mu}\{v(t+h)-v(t)\}\right\|_{p} \leq K_{2} h^{\vartheta}+K_{3} h^{1-\mu}
$$

with $\vartheta \in(0,1-\mu)$.

For the proof of (4.8), see [27, Theorem 3.1], and for (4.9), see the monograph of Rothe [21, Lemma 21].

Proof OF THEOREM 4.1. We may assume $v_{0}>0$ in $\Omega$ without loss of generality. Indeed, we can retake $v_{0}=v(T)>0$ for $T>0$ and prove this theorem with slight modification. Integrating (4.7) over [0,T] for arbitrary number $T>0$, we have

$$
\begin{aligned}
E(v(T)) & +\int_{0}^{T} \int_{\Omega} d(x) e^{\gamma(x)} \varphi^{2}\left|\nabla\left\{\log \frac{v(t)}{\varphi}\right\}\right|^{2} d x d t \\
& +\int_{0}^{T} \int_{\Omega} b(x) e^{2 \gamma(x)} \varphi\{v(t)-\varphi\}^{2} d x d t \\
& +\int_{0}^{T} \int_{\Omega} c(x) e^{2 \gamma(x)} \varphi\{v(t)-\varphi\} k *(v-\varphi)(t) d x d t \\
& =E\left(v_{0}\right)+\int_{0}^{T} \int_{t}^{\infty} k(s) d s \int_{\Omega} c(x) e^{2 \gamma(x)} \varphi^{2}\{v(t)-\varphi\} d x d t .
\end{aligned}
$$

By virtue of (2.6) and (3.2), the second term in the right hand side of (4.10) is estimated as

$$
\begin{gathered}
\int_{0}^{T} \int_{t}^{\infty} k(s) d s \int_{\Omega} c(x) e^{2 \gamma(x)} \varphi^{2}\{v(t)-\varphi\} d x d t \\
\leq\left\|c e^{2 \gamma}\right\|_{\infty} M^{2}(m+M)|\Omega| \int_{0}^{\infty} s k(s) d s .
\end{gathered}
$$

Since $t k \in L^{1}(0, \infty)$, it follows from (4.10) and the above inequality that

$$
\begin{aligned}
& \int_{0}^{T} \int_{\Omega} b(x) e^{2 \gamma(x)} \varphi\{v(t)-\varphi\}^{2} d x d t \\
& \quad+\int_{0}^{T} \int_{\Omega} c(x) e^{2 \gamma(x)} \varphi\{v(t)-\varphi\} k *(v-\varphi)(t) d x d t \leq K_{4},
\end{aligned}
$$

where

$$
K_{4}=E\left(v_{0}\right)+\left\|c e^{2 \gamma}\right\|_{\infty} M^{2}(m+M)|\Omega| \int_{0}^{\infty} t k(t) d t
$$


For $w:[0, T] \rightarrow \mathbf{R}$, define $w_{T}$ by

$$
w_{T}(t)= \begin{cases}w(t) & \text { if } t \in[0, T], \\ 0 & \text { if } t \in(-\infty, \infty) \backslash[0, T],\end{cases}
$$

and for $k:[0, \infty) \rightarrow \mathbf{R}$, define $\tilde{k}$ by

$$
\tilde{k}(t)= \begin{cases}k(t) & \text { if } t \in[0, \infty) \\ 0 & \text { if } t \in(-\infty, 0) .\end{cases}
$$

Then we can derive the following relation (cf. [27, Lemma 2.2]):

$$
\mathcal{F}\left(\tilde{k} * w_{T}\right)(\eta)=\hat{k}(i \eta) \mathcal{F} w_{T}(\eta),
$$

where $\mathcal{F} w$ denotes the Fourier transform of $w$ :

$$
\mathcal{F} w(\eta)=(2 \pi)^{-1 / 2} \int_{-\infty}^{\infty} e^{-i \eta t} w(t) d t
$$

Then we obtain

$$
\begin{aligned}
\int_{0}^{T}\left[b(x)\{v(t)-\varphi\}^{2}+c(x)\{v(t)-\varphi\} k *(v-\varphi)(t)\right] d t \\
\quad=\int_{-\infty}^{\infty}\left\{b(x)(v-\varphi)_{T}^{2}(t)+c(x)(v-\varphi)_{T}(t) \tilde{k} *(v-\varphi)_{T}(t)\right\} d t
\end{aligned}
$$

It follows from (4.12) and Parseval-Plancherel's equality that

$$
\begin{aligned}
& \int_{-\infty}^{\infty}\left\{b(x)(v-\varphi)_{T}^{2}(t)+c(x)(v-\varphi)_{T}(t) \tilde{k} *(v-\varphi)_{T}(t)\right\} d t \\
& \quad=\int_{-\infty}^{\infty}\left\{b(x)\left|\mathcal{F}(v-\varphi)_{T}(\eta)\right|^{2}+c(x) \mathcal{F}(v-\varphi)_{T}(\eta) \mathcal{F}\left(\tilde{k} *(v-\varphi)_{T}\right)(\eta)\right\} d \eta \\
& \quad=\int_{-\infty}^{\infty}\{b(x)+\operatorname{Re} \hat{k}(i \eta) c(x)\}\left|\mathcal{F}(v-\varphi)_{T}(\eta)\right|^{2} d \eta
\end{aligned}
$$

Therefore, making use of (4.1), Fubini's theorem and Parseval-Plancherel's equality again, we can obtain

$$
\begin{aligned}
& \int_{0}^{T} \int_{\Omega} b(x) e^{2 \gamma(x)} \varphi\{v(t)-\varphi\}^{2} d x d t \\
& \quad+\int_{0}^{T} \int_{\Omega} c(x) e^{2 \gamma(x)} \varphi\{v(t)-\varphi\} k *(v-\varphi)(t) d x d t \\
& \geq k_{0} \inf _{x \in \Omega}\left\{e^{2 \gamma(x)}\right\} \int_{0}^{T} \int_{\Omega}|v(t)-\varphi|^{2} \varphi d x d t .
\end{aligned}
$$


Since $T$ is arbitrary and $K_{4}$ is independent of $T$, (4.11) and (4.13) yield

$$
\varphi^{1 / 2}(v-\varphi) \in L^{1}\left((0, \infty) ; L^{2}(\Omega)\right) .
$$

On the other hand, (4.9) shows that $t \rightarrow \varphi^{1 / 2}\{v(t)-\varphi\}$ is uniformly continuous in $(0, \infty)$ with respect to $L^{2}(\Omega)$. The fact, together with (4.14) implies

$$
\lim _{t \rightarrow \infty} \int_{\Omega}|v(t)-\varphi|^{2} \varphi d x=0 .
$$

Then we can prove (4.2) from (4.15). Its proof is exactly the same as in [29] with $A$ replaced by $A+1$; so we omit it.

REMARK 4.3. Take $p>N$ and $\mu \in((p+N) /(2 p), 1)$. Then (2.1) implies

$$
\lim _{t \rightarrow \infty} v(t)=\varphi \quad \text { in } C^{1}(\bar{\Omega}) .
$$

\section{Global attractivity of $\varphi$ in case $\inf _{x \in \Omega} b(x)=0$}

In this section we consider $(\mathrm{P})$ in the case $\inf _{x \in \Omega} b(x)=0$, where one of the difficulties is to derive $L^{\infty}(\Omega)$ estimate of $v$.

THEOREM 5.1. Assume $\inf _{x \in \Omega} b(x)=0, \lambda_{1}<0$ and $t k \in L^{1}(0, \infty)$. If $\operatorname{Re} \hat{k}(i \eta) \geq 0$ for $\eta \in \mathbf{R}$, then every solution $v$ of $(\mathrm{P})$ satisfies

$$
\sup _{t \geq 0}\|v(t)\|_{\infty}<\infty .
$$

Proof. We will prove this theorem along the arguments used in [29, Proposition 3.3]. We can assume $v_{0}>0$ in $\Omega$. Since $\lambda_{1}<0$, there exists a unique positive stationary solution $\varphi$ of (SP). Then integrating (4.7) over [0,T] with any $T>0$, we see

$$
E(v(T)) \leq E\left(v_{0}\right)+\left\|c e^{\gamma}\right\|_{\infty} M \int_{0}^{T} \int_{t}^{\infty} k(s) d s \int_{\Omega} e^{\gamma(x)} \varphi v(t) d x d t
$$

as in the proof of Theorem 4.1. Since $g$ is a convex function (see (4.6)), it is possible to apply Jensen's inequality (see, e.g., [6]) to $E(v(T))$ to get

$$
\begin{aligned}
g\left(\left\|e^{\gamma / 2} \varphi\right\|_{2}^{-2} \int_{\Omega} e^{\gamma(x)} \varphi v(T) d x\right) & \leq\left\|e^{\gamma / 2} \varphi\right\|_{2}^{-2} \int_{\Omega} e^{\gamma(x)} \varphi^{2} g\left(\frac{v(T)}{\varphi}\right) d x \\
& =\left\|e^{\gamma / 2} \varphi\right\|_{2}^{-2} E(v(T)) .
\end{aligned}
$$

Put $V(t):=\left\|e^{\gamma / 2} \varphi\right\|_{2}^{-2} \int_{\Omega} e^{\gamma(x)} \varphi v(t) d x$. Then from (5.1) and (5.2)

$$
g(V(T)) \leq\left\|e^{\gamma / 2} \varphi\right\|_{2}^{-2} E\left(v_{0}\right)+\left\|c e^{\gamma}\right\|_{\infty} M \int_{0}^{T} \int_{t}^{\infty} k(s) d s V(t) d t .
$$


By using the idea in [29], it can be shown that for sufficiently large $T_{0}$,

$$
K_{6}:=\left\|c e^{\gamma}\right\|_{\infty} M \int_{T_{0}}^{\infty} \int_{t}^{\infty} k(s) d s d t<1
$$

Then (5.3) implies that for every $T \geq T_{0}$,

$$
g(V(T)) \leq\left\|e^{\gamma / 2} \varphi\right\|_{2}^{-2} E\left(v_{0}\right)+\left\|c e^{\gamma}\right\|_{\infty} M \int_{0}^{T_{0}} \int_{t}^{\infty} k(s) d s V(t) d t+K_{6} \sup _{t \in\left[T_{0}, T\right]} V(t) .
$$

Recall that $g$ is given by (4.6). Since $K_{6}<1$, it follows from the above inequality that

$$
\sup _{t \in\left[T_{0}, T\right]} V(t) \leq K_{7}
$$

with some $K_{7}$ independent of $T$. Hence it is easy to prove from (5.4) that

$$
\sup _{t \geq 0} \int_{\Omega} \varphi v(t) d x \leq K_{8}
$$

with some $K_{8}$.

Let $r \in(0,1 / 2)$. Then we can obtain from (5.5) that

$$
\sup _{t \geq 0} \int_{\Omega} v^{r}(t) d x \leq K_{9},
$$

where $K_{9}$ is a suitable positive constant independent of $t$ (for details, see [29]). Therefore, the result of Rothe [20, Proposition 2] allows us to derive the conclusion.

Repeating the arguments in the proof of Theorem 4.1, we can also obtain the following result:

THEOREM 5.2. In addition to the assumptions of Theorem 5.1, assume (4.1). Then every solution $v$ of $(\mathrm{P})$ satisfies

$$
\lim _{t \rightarrow \infty} v(t)=\varphi \quad \text { uniformly in } \bar{\Omega} .
$$

Recall that if $k$ is defined by (K.1) (resp. (K.2)), it satisfies (4.3) (resp. (4.4)). Then neither (K.1) nor (K.2) satisfies (4.1). This implies that Theorem 5.2 is inconvenient from the viewpoint of applications. By putting additional assumptions, we can improve Theorem 5.2 as follows.

THEOREM 5.3. In addition to the assumptions of Theorem 5.1, assume $k(0) \neq 0$ and $k^{\prime}(=d k / d t) \in L^{1}(0, \infty)$. Furthermore, assume that there exist positive constants $c_{0}$ and $k_{1}$ such that $c(x) \geq c_{0}$ for $x \in \Omega$ and

$$
\operatorname{Re}\{\hat{k}(i \eta)\}^{-1} \geq k_{1} \quad \text { for } \eta \in \mathbf{R} .
$$


Then every solution $v$ of $(\mathrm{P})$ satisfies

$$
\lim _{t \rightarrow \infty} v(t)=\varphi \quad \text { uniformly in } \bar{\Omega} .
$$

Proof. We will prove this theorem by the same idea as in [29, Theorem 3.5]. We may assume $v_{0}>0$ in $\Omega$. Let $\varphi$ be a unique positive stationary solution of (SP) and define a new function $w$ as

$$
w(x, t)=k *(v-\varphi)(x, t) .
$$

Similarly to the proof of Theorem 4.1, integrate (4.7) over [0,T] with an arbitrary $T>0$; then there exists a positive constant $K_{10}$, independent of $T$, such that

$$
\begin{aligned}
& E(v(T))+\int_{0}^{T} \int_{\Omega} d(x) e^{\gamma(x)} \varphi^{2}\left|\nabla\left\{\log \frac{v(t)}{\varphi}\right\}\right|^{2} d x d t \\
& \quad+\int_{0}^{T} \int_{\Omega} b(x) e^{2 \gamma(x)} \varphi\{v(t)-\varphi\}^{2} d x d t \\
& \quad+\int_{0}^{T} \int_{\Omega} c(x) e^{2 \gamma(x)} \varphi\{v(t)-\varphi\} w(t) d x d t \leq K_{10} .
\end{aligned}
$$

Since $w$ satisfies

$$
w_{t}(t)=k(0)\{v(t)-\varphi\}+k^{\prime} *(v-\varphi)(t)
$$

it follows that

$$
\begin{aligned}
\frac{1}{2} \frac{d}{d t} \int_{\Omega} c(x) e^{2 \gamma(x)} \varphi w^{2}(t) d x & =\int_{\Omega} c(x) e^{2 \gamma(x)} \varphi w(t) w_{t}(t) d x \\
& =\int_{\Omega} c(x) e^{2 \gamma(x)} \varphi w(t)\left[k(0)\{v(t)-\varphi\}+k^{\prime} *(v-\varphi)(t)\right] d x
\end{aligned}
$$

Integrate this identity over $[0, T]$ :

$$
\begin{aligned}
\frac{1}{2} \int_{0}^{T} & \frac{d}{d t} \int_{\Omega} c(x) e^{2 \gamma(x)} \varphi w^{2}(t) d x d t \\
\quad & \int_{0}^{T} \int_{\Omega} c(x) e^{2 \gamma(x)} \varphi w(t)\left[k(0)\{v(t)-\varphi\}+k^{\prime} *(v-\varphi)(t)\right] d x d t
\end{aligned}
$$

In view of $w(0)=0$,

$$
\int_{0}^{T} \frac{d}{d t} \int_{\Omega} c(x) e^{2 \gamma(x)} \varphi w^{2}(t) d x d t=\int_{\Omega} c(x) e^{2 \gamma(x)} \varphi w^{2}(T) d x \geq 0 .
$$


Therefore, we can see from (5.9)

$$
\begin{aligned}
& -\int_{0}^{T} \int_{\Omega} c(x) e^{2 \gamma(x)} \varphi w(t) k^{\prime} *(v-\varphi)(t) d x d t \\
\leq & k(0) \int_{0}^{T} \int_{\Omega} c(x) e^{2 \gamma(x)} \varphi\{v(t)-\varphi\} w(t) d x d t \\
\leq & k(0) K_{10},
\end{aligned}
$$

where we have used (5.7).

Note

$$
\begin{aligned}
\left(\hat{k}^{\prime}\right)(i \eta) & =\int_{0}^{\infty} e^{-i \eta t} k^{\prime}(t) d t \\
& =-k(0)+i \eta \hat{k}(i \eta)
\end{aligned}
$$

and $\mathcal{F}\left(w_{T}\right)(\eta)=\hat{k}(i \eta) \mathcal{F}\left((v-\varphi)_{T}\right)(\eta)$. Then

$$
\mathcal{F}\left(\tilde{k}^{\prime} *(v-\varphi)_{T}\right)(\eta)=\{-k(0)+i \eta \hat{k}(i \eta)\}\{\hat{k}(i \eta)\}^{-1} \mathcal{F}\left(w_{T}\right)(\eta) .
$$

Similarly to the proof of Theorem 4.1, we can use (5.11) and Parseval-Plancherel's equality to show

$$
\begin{aligned}
& -\int_{0}^{T} \int_{\Omega} c(x) e^{2 \gamma(x)} \varphi w(t) k^{\prime} *(v-\varphi)(t) d x d t \\
= & -\int_{\Omega} c(x) e^{2 \gamma(x)} \varphi \int_{-\infty}^{\infty} w_{T}(t) \tilde{k}^{\prime} *(v-\varphi)_{T}(t) d t d x \\
= & -\int_{\Omega} c(x) e^{2 \gamma(x)} \varphi \int_{-\infty}^{\infty} \mathcal{F}\left(w_{T}\right)(\eta) \mathcal{F}\left(\tilde{k}^{\prime} *(v-\varphi)_{T}\right)(\eta) d \eta d x \\
= & k(0) \int_{\Omega} c(x) e^{2 \gamma(x)} \varphi \int_{-\infty}^{\infty} \operatorname{Re}\{\hat{k}(i \eta)\}^{-1}\left|\mathcal{F}\left(w_{T}\right)(\eta)\right|^{2} d \eta d x .
\end{aligned}
$$

Therefore, (5.10) implies

$$
c_{0} k_{1} \inf _{x \in \Omega}\left\{e^{2 \gamma(x)}\right\} \int_{0}^{T} \int_{\Omega} \varphi w^{2}(t) d x d t \leq K_{10},
$$

where we have used (5.6). Since $T$ is arbitrary and $K_{10}$ is independent of $T$, this estimate implies

$$
\varphi^{1 / 2} w \in L^{1}\left((0, \infty) ; L^{2}(\Omega)\right)
$$

One can prove the uniformly continuity of $w(t)$ with respect to $t$ from (5.8) (see [29]). Hence it follows from (5.12) that

$$
\lim _{t \rightarrow \infty} \varphi^{1 / 2} w(t)=0 \quad \text { in } L^{2}(\Omega)
$$


In the same manner as [29] (replace $A$ by $A+1$ ),

$$
\lim _{t \rightarrow \infty} w(t)=0 \quad \text { uniformly in } \bar{\Omega} .
$$

The rest of the proof is essentially the same as [29]; so we omit it.

\section{Appendix}

Proposition A. Let $\mu_{1}^{+}$denote the positive principal eigenvalue of (3.7) with $\partial \rho / \partial n=0$ on $\partial \Omega$. Then $\mu_{1}^{+}$exists if and only if (3.10) holds true.

PROOF. We will show this proposition by using the arguments developed in the paper of Brown and Lin [7]. Note that, by virtue of (3.8), $\mu_{1}^{+}$is given by

$$
\frac{1}{\mu_{1}^{+}}=\sup _{\substack{\rho \in H^{1}(\Omega) \\ \rho \neq 0}} \frac{\int_{\Omega} a(x) e^{\gamma(x)} \rho^{2} d x}{\int_{\Omega} d(x) e^{\gamma(x)}|\nabla \rho|^{2} d x}
$$

in case $\beta(x) \equiv 0$.

1st step. We begin with the proof of the sufficiency of (3.10). If (3.10) is satisfied, then there exists a positive constant $\varepsilon_{0}$ such that

$$
\int_{\Omega} d(x) e^{\gamma(x)}|\nabla \rho|^{2} d x \geq \varepsilon_{0} \int_{\Omega} e^{\gamma(x)} \rho^{2} d x
$$

for all $\rho \in H^{1}(\Omega)$ satisfying $\int_{\Omega} a(x) e^{\gamma(x)} \rho^{2} d x>0$. We will prove this result by contradiction. If the assertion is false, then there exists a sequence $\left\{\rho_{n}\right\} \subset H^{1}(\Omega)$ such that

$$
\int_{\Omega} e^{\gamma(x)} \rho_{n}^{2} d x=1, \quad \int_{\Omega} d(x) e^{\gamma(x)}\left|\nabla \rho_{n}\right|^{2} d x \leq \frac{1}{n} \quad \text { and } \quad \int_{\Omega} a(x) e^{\gamma(x)} \rho_{n}^{2} d x>0
$$

for all $n \in \mathbb{N}$. Since $d$ is positive in $\bar{\Omega},\left\{\rho_{n}\right\}$ is bounded in $H^{1}(\Omega)$. From Rellich's theorem, one can choose a subsequence $\left\{\rho_{n^{\prime}}\right\}$ such that

$$
\lim _{n^{\prime} \rightarrow \infty} \rho_{n^{\prime}}=\rho^{*} \quad \text { weakly in } H^{1}(\Omega) \text { and strongly in } L^{2}(\Omega)
$$

with $\rho^{*} \in H^{1}(\Omega)$. From $\int_{\Omega} d(x) e^{\gamma(x)}\left|\nabla \rho_{n}\right|^{2} d x \leq 1 / n$, we see $\lim _{n \rightarrow \infty} \nabla \rho_{n}=0$ in $L^{2}(\Omega)$. Therefore, $\lim _{n^{\prime} \rightarrow \infty} \rho_{n^{\prime}}=\rho^{*}$ in $H^{1}(\Omega)$ and $\rho^{*}$ satisfies

$$
\int_{\Omega} e^{\gamma(x)}\left(\rho^{*}\right)^{2} d x=1, \quad \int_{\Omega}\left|\nabla \rho^{*}\right|^{2} d x=0 \quad \text { and } \quad \int_{\Omega} a(x) e^{\gamma(x)}\left(\rho^{*}\right)^{2} d x \geq 0 .
$$

Hence, $\rho^{*}(x) \equiv \rho_{0}$ with $\rho_{0}^{2}=1 / \int_{\Omega} e^{\gamma(x)} d x$. This fact implies

$$
\int_{\Omega} a(x) e^{\gamma(x)}\left(\rho^{*}\right)^{2} d x=\rho_{0}^{2} \int_{\Omega} a(x) e^{\gamma(x)} d x \geq 0
$$


which is a contradiction to (3.10). Thus we have shown (A.2).

We next take any $\rho \in H^{1}(\Omega)$ satisfying $\int_{\Omega} a(x) e^{\gamma(x)} \rho^{2} d x>0$. Then it follows from (A.2) that

$$
\frac{\int_{\Omega} a(x) e^{\gamma(x)} \rho^{2} d x}{\int_{\Omega} d(x) e^{\gamma(x)}|\nabla \rho|^{2} d x} \leq \frac{\int_{\Omega} a(x) e^{\gamma(x)} \rho^{2} d x}{\varepsilon_{0} \int_{\Omega} e^{\gamma(x)} \rho^{2} d x} \leq \frac{\sup _{x \in \Omega}\{a(x)\}}{\varepsilon_{0}} .
$$

Recalling (A.1) we see from (A.3) that $1 / \mu_{1}^{+} \leq \sup _{x \in \Omega}\{a(x)\} / \varepsilon_{0}$. Thus $\mu_{1}^{+}$is a positive principal eigenvalue.

2nd step. We will prove the necessity of (3.10). Let $\mu_{1}^{+}$be defined by (A.1). Assuming

$$
\int_{\Omega} a(x) e^{\gamma(x)} d x \geq 0
$$

we will derive a contradiction. If $\int_{\Omega} a(x) e^{\gamma(x)} d x>0$, then taking $\rho(x) \equiv 1$ leads us to conclude that the right-hand side of (A.1) is infinite. This fact implies the nonexistence of $\mu_{1}^{+}$, which is a contradiction.

In case $\int_{\Omega} a(x) e^{\gamma(x)} d x=0$, consider the following functional

$$
Q_{\mu}(\rho)=\int_{\Omega} d(x) e^{\gamma(x)}|\nabla \rho|^{2} d x-\mu \int_{\Omega} a(x) e^{\gamma(x)} \rho^{2} d x
$$

for each $\mu>0$. We take $\rho_{0} \in H^{1}(\Omega)$ satisfying $\int_{\Omega} a(x) e^{\gamma(x)} \rho_{0} d x>0$ (note $\rho_{0} \not \equiv$ constant). Then, for any $\delta>0$,

$$
Q_{\mu}\left(1+\delta \rho_{0}\right)=\delta^{2} Q_{\mu}\left(\rho_{0}\right)-2 \delta \mu \int_{\Omega} a(x) e^{\gamma(x)} \rho_{0} d x .
$$

Therefore, if $\delta>0$ is sufficiently small, then $\bar{\rho}_{\mu}:=1+\delta \rho_{0} \in H^{1}(\Omega)$ satisfies $Q_{\mu}\left(\bar{\rho}_{\mu}\right)<0$. This fact implies that, for any $\mu>0$, there exists $\bar{\rho}_{\mu} \in H^{1}(\Omega)$ such that

$$
\frac{1}{\mu}<\frac{\int_{\Omega} a(x) e^{\gamma(x)} \bar{\rho}_{\mu}^{2} d x}{\int_{\Omega} d(x) e^{\gamma(x)}\left|\nabla \bar{\rho}_{\mu}\right|^{2} d x} .
$$

So we see that the right-hand side of (A.1) is infinite, which is also a contradiction to the existence of positive $\mu_{1}^{+}$. Thus we have shown that (3.10) holds true.

Acknowledgment. Part of this work has been supported by Grant-in-Aid for Scientific Research (C-24540220), Japan Society for the Promotion of Science. 


\section{References}

[ 1 ] S. Ahmad and M. R. M. RaO, Stability of Volterra diffusion equations with time delays, Appl. Math. Comput. 90 (1998), 143-154.

[2] H. AmAnn, On the existence of positive solutions of nonlinear elliptic boundary value problems, Indiana Univ. Math. J. 21 (1971), 125-146.

[3] F. Belgacem and C. COSNER, The effects of dispersal along environmental gradients on the dynamics of populations in heterogeneous environments, Canad. Appl. Math. Quart. 3 (1995), 379-397.

[ 4 ] H. BERESTYCKI, Le nombre de solutions de certains problèmes semi-linéaires elliptiques, J. Funct. Anal. 40 (1981), 1-29.

[5] A. Bezuglyy and Y. Lou, Reaction-diffusion models with large advection coefficients, Appl. Anal. 89 (2010), 983-1004.

[6] H. BrezIS, Functional Analysis, Sobolev Spaces and Partial Differential Equations, Universitext, SpringerVerlag, New York, 2011.

[ 7 ] K. J. BROWN and S. S. LIN, On the existence of positive eigenfunctions for an eignevalue problem with indefinite weight function, J. Math. Anal. Appl. 75 (1980), 112-120.

[ 8 ] R. F. CANTRELl and C. Cosner, Diffusive logistic equations with indefinite weights: population models in disrupted environments, Proc. Roy. Soc. Edinburgh Sect. A 112 (1989), 293-318.

[ 9 ] R. F. CAntrell and C. Cosner, The effects of spatial heterogeneity in population dynamics, J. Math. Biol. 29 (1991), 315-338.

[10] R. F. Cantrell and C. Cosner, Spatial Ecology via Reaction-Diffusion Equations, John Wiley \& Sons, Ltd., Chichester, 2003.

[11] C. Cosner and Y. Lou, Does movement toward better environments always benefit a population?, J. Math. Anal. Appl. 277 (2003), 489-503.

[12] D. Henry, Geometric Theory of Semilinear Parabolic Equations, Lecture Notes in Mathematics Vol. 840, Springer-Verlag, Berlin-New York, 1981.

[13] H. Hoshino and Y. YAmada, Solvability and smoothing effect for semilinear parabolic equations, Funkcial. Ekvac. 34 (1991), 475-494.

[14] M. IIDA, Exponentially asymptotic stability for a certain class of semilinear Volterra diffusion equations, Osaka J. Math. 28 (1991), 411-440.

[15] K-Y. LAM, Concentration phenomena of a semilinear elliptic equation with large advection in an ecological model, J. Differential Equations 250 (2011), 161-181.

[16] H. LiU and H. YU, Entropy/energy stable schemes for evolutionary dispersal models, J. Comput. Phys. 256 (2014), 656-677.

[17] A. PAZY, Semigroups of Linear Operators and Applications to Partial Differential Equations, Applied Mathematical Sciences Vol. 44, Springer-Verlag, New York, 1983.

[18] M. H. Protter and H. F. Weinberger, Maximum Principles in Differential Equations, Springer-Verlag, New York, 1984.

[19] R. Redlinger, On Volterra's population equation with diffusion, SIAM J. Math. Anal. 16 (1985), 135-142.

[20] F. ROTHE, Uniform bounds from bounded $L_{p}$-functionals in reaction-diffusion equations, J. Differential Equations 45 (1982), 207-233.

[21] F. Rothe, Global Solutions of Reaction-Diffusion Systems, Lecture Notes in Mathematics Vol. 1072, SpringerVerlag, Berlin, 1984.

[22] D. H. SATtinger, Monotone methods in nonlinear elliptic and parabolic boundary value problems, Indiana Univ. Math. J. 21 (1972), 979-1000.

[23] A. Schiaffino, On a diffusion Volterra equation, Nonlinear Anal. 3 (1979), 595-600.

[24] A. Schiaffino and A. Tesei, Monotone methods and attractivity results for Volterra integro-partial differential equations, Proc. Roy. Soc. Edinburgh Sect. A 89 (1981), 135-142. 
[25] J. Smoller, Shock Waves and Reaction-Diffusion Equations, Second edition, Grundlehren der Mathematischen Wissenschaften Vol. 258, Springer-Verlag, New York, 1994.

[26] A. TeseI, Stability properties for partial Volterra integro-differential equations, Ann. Mat. Pura Appl. 126 (1980), 103-115.

[27] Y. YAmadA, On a certain class of semilinear Volterra diffusion equations, J. Math. Anal. Appl. 88 (1982), 433-457.

[28] Y. YAmADA, Asymptotic stability for some systems of semilinear Volterra diffusion equations, J. Differential Equations 52 (1984), 295-326.

[29] Y. YAMADA, Asymptotic behavior of solutions for semilinear Volterra diffusion equations, Nonlinear Anal. 21 (1993), 227-239.

\author{
Present Addresses: \\ YUSUKE YOSHIDA \\ DePartment of PuRE And Applied Mathematics, \\ WASEDA UNIVERSITY, \\ 3-4-1 OHKUBo, SHINJUKU-KU, TOKYo 169-8555, JAPAN. \\ e-mail: ypau-yoshida@suou.waseda.jp \\ Yoshio YAMADA \\ DEPARTMENT OF APPLIED MATHEMATICS, \\ WASEDA UNIVERSITY, \\ 3-4-1 OHкUBo, SHINJUKU-KU, TOKYo 169-8555, JAPAN. \\ e-mail: yamada@waseda.jp
}

